apical $\mathrm{ENaC}$ abundance largely through signaling mechanisms that do not require Nedd4-2. This conclusion derives not only from the current paper (8), but also from work showing that aldosterone responsiveness of $\mathrm{ENaC}$ in the rectum is increased rather than decreased when Nedd4-2 is deleted (18) and that aldosterone still regulates $\mathrm{ENaC}$ trafficking in the kidney when PY motifs are deleted in vivo (4). One possibility, suggested by the current results, is that Nedd4-2-mediated ubiquitylation primarily accelerates $\mathrm{ENaC}$ degradation within the cell (step iii in Figure 1A) rather than its removal from the membrane (step ii in Figure 1A). Secondly, Nedd4-2 appears to play a physiologically significant and nonredundant role in modulating NCC abundance. That said, it should be pointed out that SGK1 also activates NCC through pathways independently of Nedd4-2 (16) and that other NCC modulators, such as WNK4, may also have large effects on NCC activity. Finally, this work shows again why it is so important to test mechanistic models in vivo. While the results are sometimes confusing and can challenge accepted dogma, their relevance to human health and disease is beyond question. An intriguing clinical implication of the paper by Ronzaud and colleagues (8) is that manipulating Nedd4-2 activity might be a novel approach to treating Gitelman syndrome, an ionic imbalance disease linked to reductions in membrane NCC (19). As Nedd4-2 may inhibit both membrane removal and intracellular deg- radation, its inhibition might increase NCC abundance enough to ameliorate the troubling symptoms of the disease.

\section{Acknowledgments}

The author acknowledges the review and suggestions provided by James McCormick. The author's laboratory is supported by funds from the Department of Veterans Affairs and the NIH (DK 5 RO1 51496 and DK 1 RO1 095841).

Address correspondence to: David H. Ellison, Division of Nephrology and Hypertension, CH12R, Oregon Health and Science University, 3181 SW Sam Jackson Park Road, Portland, Oregon 97239, USA. Phone: 503.494.8490; Fax: 503.494.5330; E-mail: ellisond@ohsu.edu.

1. Staub O, et al. WW domains of Nedd4 bind to the proline-rich PY motifs in the epithelial $\mathrm{Na}^{+}$ channel deleted in Liddle's syndrome. $E M B O J$. 1996;15(10):2371-2380.

2. Rotin D. Role of the UPS in Liddle syndrome. BMC Biochem. 2008;9(suppl 1):S5.

3. Abriel H, et al. Defective regulation of the epithelial $\mathrm{Na}+$ channel by Nedd 4 in Liddle's syndrome. J Clin Invest. 1999;103(5):667-673.

4. Pradervand S, et al. Dysfunction of the epithelial sodium channel expressed in the kidney of a mouse model for Liddle syndrome. J Am Soc Nephrol. 2003;14(9):2219-2228.

5. Ko B, Kamsteeg EJ, Cooke LL, Moddes LN, Deen PM, Hoover RS. RasGRP1 stimulation enhances ubiquitination and endocytosis of the sodiumchloride cotransporter. Am J Physiol Renal Physiol. 2010;299(2):F300-F309.

6. Arroyo JP, et al. Nedd4-2 modulates renal Na+-Clcotransporter via the aldosterone-SGK1-Nedd4-2 pathway. J Am Soc Nephrol. 2011;22(9):1707-1719.
7. McCormick JA, Bhalla V, Pao AC, Pearce D. SGK1: a rapid aldosterone-induced regulator of renal sodium reabsorption. Physiology (Bethesda). 2005;20:134-139.

8. Ronzaud C, et al. Renal tubular NEDD4-2 deficiency causes NCC-mediated salt-dependent hypertension. J Clin Invest. 2013;123(2):657-665.

9. Traykova-Brauch $\mathrm{M}$, et al. An efficient and versatile system for acute and chronic modulation of renal tubular function in transgenic mice. Nat Med. 2008;14(9):979-984.

10. Shi PP, et al. Salt-sensitive hypertension and cardiac hypertrophy in mice deficient in the ubiquitin ligase Nedd4-2. Am J Physiol Renal Physiol. 2008; 295(2):F462-F470.

11. Boase NA, et al. Respiratory distress and perinatal lethality in Nedd4-2-deficient mice. Nat Commun. 2011;2:287.

12. Louis-Dit-Picard H, et al. KLHL3 mutations cause familial hyperkalemic hypertension by impairing ion transport in the distal nephron. Nat Genet. 2012;44(4):456-460.

13. Boyden LM, et al. Mutations in kelch-like 3 and cullin 3 cause hypertension and electrolyte abnormalities. Nature. 2012;482(7383):98-102.

14. Metzger MB, Hristova VA, Weissman AM. HECT and RING finger families of E3 ubiquitin ligases at a glance. J Cell Sci. 2012;125(pt 3):531-537.

15. Faresse $\mathrm{N}$, et al. Inducible kidney-specific Sgk1 knockout mice show a salt-losing phenotype. American journal of physiology. Am J Physiol Renal Physiol. 2012;302(8):F977-F985.

16. Rozansky DJ, et al. Aldosterone mediates activation of the thiazide-sensitive $\mathrm{Na}-\mathrm{Cl}$ cotransporter through an SGK1 and WNK4 signaling pathway. J Clin Invest. 2009;119(9):2601-2612.

17. Loffing-Cueni D, et al. Dietary sodium intake regulates the ubiquitin-protein ligase nedd4-2 in the renal collecting system. J Am Soc Nephrol. 2006;17(5):1264-1274.

18. Bertog $\mathrm{M}$, et al. Aldosterone responsiveness of the epithelial sodium channel $(\mathrm{ENaC})$ in colon is increased in a mouse model for Liddle's syndrome. J Physiol. 2008;586(2):459-475.

19. Kunchaparty S, et al. Defective processing and expression of the thiazide-sensitive $\mathrm{Na}-\mathrm{Cl}$ cotransporter as a cause Gitelman's Syndrome. Am J Physiol Renal Physiol. 1999;277(4 pt 2):F643-F649.

\title{
Multiple functions of a glioblastoma fusion oncogene
}

\author{
Ivan Babic and Paul S. Mischel
}

Ludwig Institute for Cancer Research, UCSD, La Jolla, California, USA.

\begin{abstract}
RNA sequencing facilitates the discovery of novel gene fusions in cancer. In this issue of the JCI, Parker et al. identify an FGFR3-TACC3 fusion oncogene in glioblastoma and demonstrate a novel mechanism of pathogenicity. A miR-99a binding site within the $3^{\prime}$-untranslated region ( 3 'UTR) of FGFR3 is lost, releasing FGFR3 signaling from miR-99a-dependent inhibition and greatly enhancing tumor progression relative to WT FGFR3. These results provide compelling insight into the pathogenicity of a novel fusion oncogene and suggest new therapeutic approaches for a subset of glioblastomas.
\end{abstract}

Conflict of interest: The authors have declared that no conflict of interest exists.

Citation for this article: J Clin Invest. 2013; 123(2):548-551. doi:10.1172/JCI67658.
Genomic technologies are transforming our knowledge about the mutational landscape of cancer. For glioblastoma, the most common and lethal form of adult primary brain cancer, integrated DNA, transcriptional, and epigenetic analyses have identified copy number alterations, mutations, tumor transcriptional and epigenetic subclasses, and potential new drug targets (1-5). Recent progress in next-generation sequencing technologies, including RNA sequencing, provides a powerful new platform adding to this integrated toolkit. Researchers are now able to identify and quantify changes in both coding and noncoding RNA; identify alternative splicing events; and detect expressed mutations, SNPs, gene 


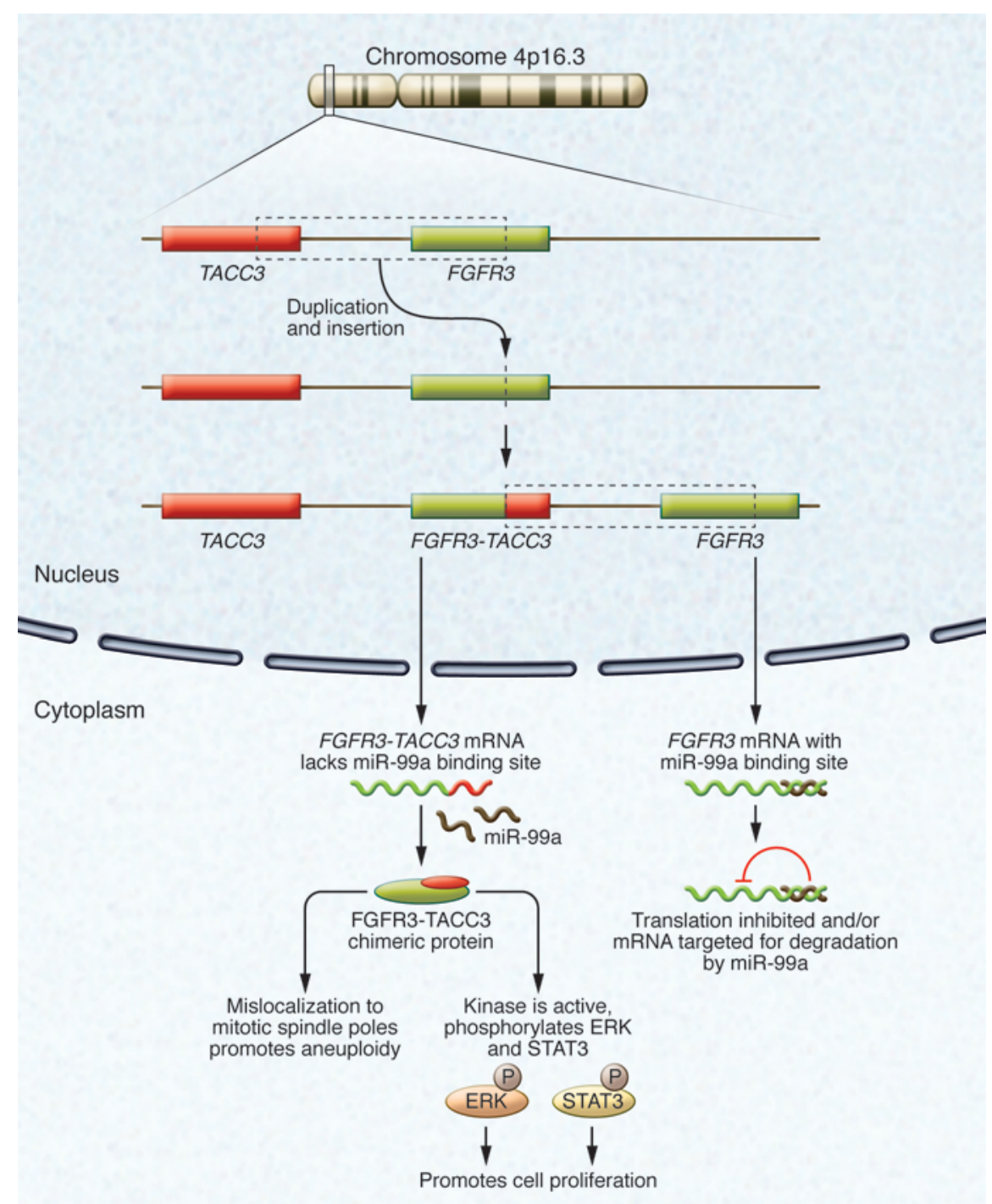

translocations, and fusion transcripts. Importantly, the identification of novel fusion proteins may provide new insights into the biology of this dreadful disease.

\section{Gene fusions and cancer}

Fusion genes occur when parts of two genes combine during a chromosomal rearrangement, resulting in expression of a chimeric protein, a process whose importance in cancer is well recognized. $B C R-A B L 1$ in chronic myelogenous leukemia (CML; ref. 6), PML-RARA in acute promyelocytic leukemia (APL; reviewed in refs. 7, 8), EML4-Alk in non-small-cell lung cancer (NSCLC; reviewed in ref. 9), EWS-FLI in Ewing sarcoma (10), and TMPRSS2-ERG in prostate cancer (11) are paradigmatic examples. Fusion oncogenes are not common in cancer in gen- eral, but their importance in understanding cancer biology is disproportionately large, providing some of the most compelling examples of successful targeted therapies for selected cancer subtypes. Mechanistic insights gleaned by studying the BCR-ABL1 and PML-RARA oncogenes have translated into near-cures for two previously deadly types of cancer: imatinib for the treatment of CML and combined arsenic trioxide and retinoic acid for the treatment of APL $(6,7)$. Of note, the finding of EML4-Alk fusion and its rapid translation into clinical benefit for NSCLC patients treated with crizotinib brings new hope that the insights gained from studying fusions may not be limited to the rarer types of molecularly homogeneous hematopoietic cancers, such as CML and APL. Thus, discovery of

\section{Figure 1}

A tandem duplication event results in the formation of an FGFR3-TACC3 fusion product. Here, Parker et al. demonstrate that the fusion transcript lacks a miR-99a binding site, resulting in increased expression (12). In addition, FGFR3-TACC3 fusion activates ERK and STAT3 signaling and enhances tumor progression. Previous work also demonstrated that localization of this fusion protein to the mitotic spindles promotes aneuploidy (13). a new fusion in glioblastoma is an exciting and important development.

\section{FGFR3-TACC3 gene fusion in glioblastoma}

In this issue, Parker et al. used whole-transcriptome RNA sequencing (RNA-seq) to look for fusions formed as a result of chromosomal translocations (12). Analysis of 48 glioblastoma samples and 43 low-grade glioma samples obtained from the United States and China, as well as analysis of the large Cancer Genome Atlas dataset, revealed the presence of a fusion composed of FGF receptor 3 (FGFR3) with transforming acidic coiled-coil 3 (TACC 3). The authors demonstrated that fusion occurred via a tandem duplication event and detected it in $8.3 \%$ of glioblastoma patients. It was not detected in any 
of the low grade glioma samples. Importantly, FGFR3-TACC3 fusions were mutually exclusive with EGFR, PDGFR, or MET genetic alterations, the receptor tyrosine kinase alterations commonly detected in glioblastoma. Taken together, these findings suggest an important and specific role for FGFR3-TACC3 in promoting glioblastoma growth.

This study by Parker and colleagues independently validates the very recent report by Singh et al. (13), which identified FGFR3-TACC fusions in a small subset of glioblastomas. While independent validation is essential, the real excitement of this study lies more in its identification of the novel and potentially targetable mechanism of pathogenicity created by the FGFR3-TACC3 fusion.

FGFR3 encodes a receptor tyrosine kinase that is commonly mutated in bladder and cervical cancer (14). FGFR3 engages downstream signaling cascades that are commonly activated in cancer, including PI3K-Akt and Ras-Mek-Erk signaling (15). In glioblastoma, a role for FGFR3 had not been previously established, although recent work suggests that it can phosphorylate PTEN to promote glioblastoma resistance to EGFR tyrosine kinase inhibitors (16). TACC3 encodes a centrosomal protein involved in mitosis (17) that is overexpressed in lung and colon carcinomas and in multiple myeloma (18). What are the mechanisms of its pathogenicity, and in what ways are the activities of this fusion protein greater than the sum of its parts?

Singh et al. demonstrated that the transforming capacity of FGFR3-TACC3 is related to its localization to the mitotic spindle, where it causes chromosomal missegregation and aneuploidy. Importantly, they showed that this requires FGFR kinase activity, because a pan-FGFR tyrosine kinase inhibitor abrogated the fusion protein's effects on chromosomal instability, reversing aneuploidy (13). Thus, Singh and colleagues identified one intriguing mechanism by which FGFR3-TACC3 fusion can promote tumorigenicity.

In the present study, Parker and colleagues provide an alternative and entirely new view of a mechanism driving FGFR3-TACC3 pathogenicity (Figure 1). They conclude that the pathogenicity of FGFR3-TACC3 is mediated, at least in part, through loss of the miR-99a binding site. MicroRNAs typically bind the $3^{\prime}$-untranslated region (3'-UTR) of a tran- script and can repress translation and/or promote degradation of that transcript (19). Changes in the 3'-UTR, due to alternative splicing or shortening through alternative cleavage, can significantly affect mRNA translation $(20,21)$, resulting in enhanced expression of transcripts insensitive to microRNA regulation. This may promote tumor development and/ or progression $(20,21)$. Parker and colleagues demonstrated that a miR-99a binding site in the 3 '-UTR of FGFR3 was lost during fusion of FGFR3 with TACC3, causing greatly increased FGFR3 expression, an effect that was counteracted by reintroduction of the $3^{\prime}$-UTR of FGFR3 in the presence of miR-99a (12). Importantly, FGFR3-TACC3 fusion was demonstrated to preferentially engage ERK and STAT3 signaling and to enhance tumor progression in vivo relative to WT FGFR3, which suggests that the fusion creates a specific gain of function.

\section{New approaches}

Glioblastoma is now one of the most intensely studied of all cancers at the molecular level; however, the mapping of the mutational landscape has yet to be successfully leveraged to yield better treatment for patients. Obtaining a mechanistic understanding of this mutational landscape, particularly with regard to clarifying function of specific mutations, may go a long way toward transforming basic science knowledge into clinical benefit for patients. Here, Parker and colleagues have taken important steps toward developing a functional understanding of the consequences of the FGFR3-TACC 3 fusion in glioblastoma (12). Their study, taken together with the work of Singh et al. (13), demonstrates the importance of the FGFR3-TACC3 fusion in glioblastoma, which suggests that the gain of function created by the FGFR3-TACC3 fusion is greater than the sum of its parts.

The current study also raises several questions for further investigation. First, what are the mechanisms by which the FGFR3 component of the fusion protein differentially engages downstream signaling, enabling it to more effectively activate the ERK and STAT3 signaling cascades? Second, the data from Singh et al. suggest a critical role for the TACC3 component of the fusion protein in regulating aneuploidy in a fashion dependent on FGFR3 signaling; how is that regulated? Finally, what are the therapeutic implications? Will glioblastomas bearing FGFR3-TACC3 fusions respond to pharmacologic inhibition with FGFR3 inhibitors, when they become available? Efforts to elucidate the mechanisms by which fusion oncogenes promote their oncogenic effects, even if they are rare, have yielded remarkable therapeutic insights. We expect that similar deep investigation into these pathogenic mechanisms, and others that will be discovered through RNA sequencing, will also reveal new therapeutic strategies.

\section{Acknowledgments}

This work is supported by grants from National Institute for Neurological Diseases and Stroke (NS73831), the National Cancer Institute (CA119347), and the Ben and Catherine Ivy Foundation.

Address correspondence to: Paul S. Mischel, Ludwig Institute for Cancer Research, University of California at San Diego, La Jolla, California 92093, USA. Phone: 858.534.6080; Fax: 858.534.7750; E-mail: pmischel@ucsd.edu.

1. Cancer Genome Atlas Research Network. Comprehensive genomic characterization defines human glioblastoma genes and core pathways. Nature. 2008;455(7216):1061-1068.

2. Parsons DW, et al. An integrated genomic analysis of human glioblastoma multiforme. Science. 2008;321(5897):1807-1812.

3. Verhaak RG, et al. Integrated genomic analysis identifies clinically relevant subtypes of glioblastoma characterized by abnormalities in PDGFRA, IDH1, EGFR, and NF1. Cancer Cell. 2010; 17(1):98-110.

4. Noushmehr $\mathrm{H}$, et al. Identification of a CpG island methylator phenotype that defines a distinct subgroup of glioma. Cancer Cell. 2010;17(5):510-522.

5. Sturm D, et al. Hotspot mutations in H3F3A and IDH1 define distinct epigenetic and biological subgroups of glioblastoma. Cancer Cell. 2012;22(4):425-437.

6. Perrotti D, Jamieson C, Goldman J, Skorski T. Chronic myeloid leukemia: mechanisms of blastic transformation. J Clin Invest. 2010; 120(7):2254-2264.

7. Lallemand-Breitenbach V, Zhu J, Chen Z, de Thé H. Curing APL through PML/RARA degradation by As2O3. Trends Mol Med. 2012;18(1):36-42.

8. Soda M, et al. Identification of the transforming EML4-ALK fusion gene in non-small-cell lung cancer. Nature. 2007;448(7153):561-566.

9. Gerber DE, Minna JD. ALK inhibition for nonsmall cell lung cancer: from discovery to therapy in record time. Cancer Cell. 2010;18(6):548-551.

10. Lessnick SL, Ladanyi M. Molecular pathogenesis of Ewing sarcoma: new therapeutic and transcriptional targets. Annu Rev Pathol. 2012;7:145-159.

11. Prensner JR, Chinnaiyan AM. Oncogenic gene fusions in epithelial carcinomas. Curr Opin Genet Dev. 2009;19(1):82-91.

12. Parker BC, et al. The tumorigenic FGFR3-TACC3 gene fusion escapes miR-99a regulation in glioblastoma. J Clin Invest. 2013;123(2):855-865.

13. Singh D, et al. Transforming fusions of FGFR 
and TACC genes in human glioblastoma. Science. 2012;337(6099):1231-1235

14. Cappellen D, et al. Frequent activating mutations of FGFR3 in human bladder and cervix carcinomas. Nat Genet. 1999;23(1):18-20.

15. Eswarakumar VP, Lax I, Schlessinger J. Cellular signaling by fibroblast growth factor receptors. Cytokine Growth Factor Rev. 2005;16(2):139-149.

16. Fenton TR, et al. Resistance to EGF receptor inhibitors in glioblastoma mediated by phosphorylation of the PTEN tumor suppressor at tyrosine 240. Proc
Natl Acad Sci U S A. 2012;109(35):14164-14169. 17. Gergely F, Kidd D, Jeffers K, Wakefield JG, Raff JW. D-TACC: a novel centrosomal protein required for normal spindle function in the early Drosophila embryo. EMBO J. 2000;19(2):241-252.

18. Still IH, Vince P, Cowell JK. The third member of the transforming acidic coiled coil-containing gene family, TACC 3 , maps in $4 \mathrm{p} 16$, close to translocation breakpoints in multiple myeloma, and is upregulated in various cancer cell lines. Genomics. 1999;58(2):165-170.
19. Fabian MR, Sonenberg N, Filipowicz W. Regulation of mRNA translation and stability by microRNAs. Annu Rev Biochem. 2010;79:351-379.

20. Mayr C, Bartel DP. Widespread shortening of 3'UTRs by alternative cleavage and polyadenylation activates oncogenes in cancer cells. Cell. 2009; 138(4):673-684.

21. Sandberg R, Neilson JR, Sarma A, Sharp PA, Burge CB. Proliferating cells express mRNAs with shortened 3' untranslated regions and fewer microRNA target sites. Science. 2008;320(5883):1643-1647.

\title{
Old King Coal - molecular mechanisms underlying an ancient treatment for atopic eczema
}

\author{
W.H. Irwin McLean' and Alan D. Irvine ${ }^{2,3,4}$
}

\begin{abstract}
${ }^{1}$ Centre for Dermatology and Genetic Medicine, Division of Molecular Medicine, Colleges of Life Sciences and Medicine, Dentistry and Nursing, University of Dundee, Dundee, United Kingdom. '2Department of Paediatric Dermatology, Our Lady's Children's Hospital, Dublin, United Kingdom. ${ }^{3}$ Clinical Medicine, Trinity College Dublin, Dublin, United Kingdom. ${ }^{4}$ National Children's Research Centre,

Our Lady's Children's Hospital, Dublin, United Kingdom.
\end{abstract}

\begin{abstract}
Traditional remedies for common disorders have been known for centuries, but insight into their mechanism of action is often limited. In this issue of the JCI, Joost Schalkwijk's research group at the Radboud University Nijmegen Medical Centre in The Netherlands advances our understanding of why topical coal tar is an effective treatment for atopic dermatitis (AD), both rationalizing the use of this traditional medicine, and providing the scientific basis for new therapeutic approaches.
\end{abstract}

$\mathrm{AD}$ (also called "eczema") is the most common inflammatory skin condition, affecting about $20 \%$ of children in the developed world. AD is a classic complex trait where a combination of several genetic predisposing factors interact with environmental stimuli to trigger the disease. AD is frequently associated with high serum $\operatorname{IgE}$ and a Th2 immune response $(1,2)$. In 2006, a paradigm shift in the pathomechanistic understanding of $\mathrm{AD}$ took place when loss-of-function mutations were discovered in the FLG gene encoding the skin barrier protein filaggrin in the common monogenic skin disease ichthyosis vulgaris (dry, flaky skin) (3). Soon thereafter, these same filaggrin variants, which are carried by about $10 \%$ of populations of European ancestry and persist at high frequencies in other populations (4), were shown to be the major genetic predisposing factor in $\mathrm{AD}$ (5). Filaggrin-deficient animals were subsequently shown to have a "leaky" skin bar-

Conflict of interest: The authors have declared that no conflict of interest exists.

Citation for this article: J Clin Invest. 2013; 123(2):551-553. doi:10.1172/JCI67438. rier, allowing passive percutaneous transfer of antigens, which trigger skin inflammation and an allergic immune response, analogous to AD in humans (6). This work showed that a primary skin barrier deficiency is at least one important factor in $\mathrm{AD}$ pathogenesis, although Th2 immunity is clearly also a major player (1).

\section{Making the pitch for pitch}

Coal tar has been used medically since ancient times. In his epic 5-volume work,

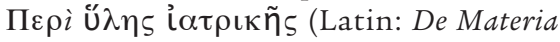
Medica), the Greek physician, pharmacologist, and botanist Pedanius Dioscorides (circa 40-90 CE) chronicled many and varied herbal and other remedies in use at the time, including the "grime of a gymnasium wall." While this remedy for abrasions and ulcers does not currently enjoy popularity, his suggestion of the use of bitumen or asphalt/coal tar for "inflammation" (7) has maintained traction over the succeeding 2 millennia (8). For as long as modern dermatology departments have existed, liquor picis carbonis (LPC) has been a part of their working vocabulary, and preparations containing LPC are widely considered to be effective in the treatment of psoriasis and AD. Indeed, a recent systematic review provided evidence of the efficacy of $0.5 \%-5 \%$ LPC preparations for both these conditions (9).

Tar, asphalt, bitumen, and pitch are related substances consisting of complex mixtures of high molecular weight organic compounds that can be derived from heat distillation of plants, wood, petrochemicals, or coal. Pitch essentially functions as a solid but is really an incredibly viscous liquid that is estimated to have more than 100 billion times the viscosity of water (10). The world's longest continuously running laboratory experiment is The University of Queensland's "Pitch Drop Experiment" (10). Begun in 1927-1930 by Thomas Parnell (it took 3 years just for the pitch to settle into a glass funnel), droplets of pitch fall under gravity only about once a decade, with the next one expected this year. We live in exciting times!

\section{A target identified}

Although the terms are somewhat ambiguous and interchangeable, pitch tends to refer to the more solid substances in this group, and tar generally refers to the more liquefied products. Coal tar is an extremely viscous liquid obtained from dry-heating coal to temperatures in the range of $900^{\circ} \mathrm{C}$ to $1200^{\circ} \mathrm{C}$, and is thought to consist of at least 10,000 distinct high molecular weight hydrocarbon and aromatic compounds, 